\title{
Video Article \\ Modified MicroSecure Vitrification: A Safe, Simple and Highly Effective Cryopreservation Procedure for Human Blastocysts
}

\author{
Mitchel C. Schiewe ${ }^{1}$, Shane Zozula ${ }^{1}$, Nancy Nugent ${ }^{1}$, Kelley Waggoner ${ }^{1}$, Jessica Borba ${ }^{1}$, Lisa Gamboa ${ }^{1}$, John B. Whitney ${ }^{1}$ \\ ${ }^{1}$ Ovation Fertility
}

Correspondence to: Mitchel C. Schiewe at mcschiewe@verizon.net

URL: https://www.jove.com/video/54871

DOI: doi: $10.3791 / 54871$

Keywords: Developmental Biology, Issue 121, blastocysts, cryopreservation, vitrification, infertility, quality control, aseptic storage

Date Published: 3/2/2017

Citation: Schiewe, M.C., Zozula, S., Nugent, N., Waggoner, K., Borba, J., Gamboa, L., Whitney, J.B. Modified MicroSecure Vitrification: A Safe, Simple and Highly Effective Cryopreservation Procedure for Human Blastocysts. J. Vis. Exp. (121), e54871, doi:10.3791/54871 (2017).

\section{Abstract}

Clinical embryo vitrification evolved with the development of unique vitrification devices in the $21^{\text {st }}$ century and with the misconception that ultra-rapid cooling in an "open" system (i.e., direct $\mathrm{LN}_{2}$ contact) was a necessity to optimize vitrification success. The dogma surrounding the importance of cooling rates led to unsafe practices subject to technical variation and to the creation of vitrification devices that disregarded important quality-control factors (e.g., ease of use, repeatability, reliability, labeling security, and storage safety). Understanding the qualitycontrol flaws of other devices allowed for the development of a safe, secure, repeatable, and reliable $\mu S-V T F$ method aimed to minimize intraand inter-technician variation. Equally important, it combined the availability of two existing FDA-compliant devices: 1) a 0.3-mL ionomeric resin embryo straw with internalized, dual-colored, tamper-proof labeling with repeatable weld seal potential; and 2) shortened, commonly-used, $300-\mu \mathrm{m}$ ID sterile flexipettes to directly load the embryo(s) in order to create a highly-effective global vitrification device. Like other aseptic, closed vitrification systems (e.g., High Security Vitrification (HSV), Rapid-i, and VitriSafe) effectively used in reproductive medicine, microSecure Vitrification ( $\mu$ S-VTF) has proven that it can achieve high post-warming survival and pregnancy outcomes with its attention to simplicity, and reduced technical variation. Although the $0.3-\mathrm{mL}$ embryo straw containing an internal hydrophobic plug was commercially replaced with a standard semen straw possessing cotton-polyvinyl pyrrolidone (PVP) plugs, it maintained its ionomeric resin composition to ensure weld sealing. However, the cotton plugs can wick out the fluid-embryo contents of the flexipettes upon contact. A modified $\mu S$-VTF method was adapted to include an additional internal weld seal before the plug on the device loading side. The added technical step to the $\mu$ S-VTF procedure has not affected its successful application, as high survival rates (>95\%) and pregnancy rates continue today.

\section{Video Link}

The video component of this article can be found at https://www.jove.com/video/54871/

\section{Introduction}

Vitrification is the single most impactful assisted reproductive technology in the in vitro fertilization (IVF) industry since the development of intracytoplasmic sperm injection. Today, blastocysts are cryopreserved without the loss in embryo viability previously associated with conventional slow-freezing methods ${ }^{1}$. With reliable post-warming embryo survival, the infertility industry is transforming into the preferred use of cryopreserved embryo transfer cycles, which yield similar or higher pregnancy outcomes than traditional fresh embryo transfer. In association with blastocyst biopsy and preimplantation genetic screening (PGS), vitrification has become a vital clinical tool to optimize healthy live birth outcomes via euploid single embryo transfer ${ }^{2,3}$.

Murine embryo vitrification was developed in the mid- $1980 \mathrm{~s}^{4,5}$ and adapted to animal agriculture by $1990^{6}$. Based on the premise that vitrification solutions form a metastable glasseous state, free of damaging ice crystal formation, it has proven to more efficiently preserve the complete cellular integrity of embryos. Interestingly, the promising acceptance of vitrification into human embryology did not begin to be realized until the $21^{\text {st }}$ century. Early publications promoting the use of vitrification coincided with the development of unique "open" system devices ${ }^{7,8,9}$. However, the adoption of vitrification into clinical practice was slow, as it came at a time when improvements in slow blastocyst freezing were also occurring. Successful conventional slow-rate freezing, in addition to vitrification, were aligned with improvements in embryo culture systems, as well as with the incorporation of blastocoele-collapsing approaches, which enhanced both the overall survival of trophectoderm and, subsequently, implantation ${ }^{10}$.

In the last decade, vitrification technology has rapidly supplanted conventional freezing practices. To a great extent, this was due to the development of specialized vitrification devices. Some of these devices have handicapped the overall safety, efficiency and effectiveness of clinical vitrification by introducing inherent design flaws to devices used in the IVF industry ${ }^{11}$. Indeed, the nuances of different devices introduce significant technical variation between programs, commonly referred to as "technical signatures"12. Thus, scientific journals, like the Journal of Visualized Experiments (JoVE), can serve as a valuable resource for demonstrating technical details, which will help to reduce outcome variation. Another ongoing problem is that some embryologists continue to be misinformed, even today, based on claims that the "ultra-rapid 
cooling of embryos or oocytes in an 'open vitrification system' (i.e., direct embryo contact with liquid nitrogen (LN $\left.\mathrm{LN}_{2}\right)$ ) is a prerequisite to optimizing success rates." Clearly, this belief is inaccurate, based on the proven success of aseptic closed systems ${ }^{13,14,15}$.

Based on the cryobiological principles of vitrification, the efficacy of vitrification is more highly dependent upon warming rates than on cooling rates $^{16,17,18}$. In general, independent of the vitrification device used, the warming rate must exceed the cooling rate to insure high survival rates. High warming rates minimize the opportunity for any ice growth (i.e., the recrystallization of nucleated impurities in cryo-solutions) during the devitrification phase of warming. Granted, the stability of the vitrification solution (i.e., the type and concentration of cryoprotective agents used) may have a confounding effect, but this is addressed in a separate publication ${ }^{11}$. Considering the cooling-warming rate issues, MicroSecure Vitrification ( $\mu \mathrm{S}-\mathrm{VTF}$ ) was developed in 2008 as an inexpensive, non-commercial, FDA-compliant method that optimized the quality-control aspects of vitrification. It was unique in that it offered tamper-proof, internalized, dual-colored labeling. Furthermore, by loading and storing the embryos directly in the sterile flexipette used for pipetting (i.e., without pipetting to a secondary device surface) and by using ionomeric-resin straws that completely weld seal using an automated sealer, technical variation has been effectively eliminated.

When assessing the completeness of vitrification devices for potential use, there are several quality-control factors that should be taken into account, including: 1) Labeling potential-Can labels be securely adhered? Are they tamperproof? Do they offer dual-color identification potential? Does it require a secondary label, and can the label be easily removed for record-keeping purposes (i.e., patient verification) postwarming? 2) Technical ease-Can embryos be easily loaded into/onto the device in a timely manner and simply identified and tracked postwarming? 3) Procedural simplicity/Repeatability-Does the vitrification method offer simplicity and reliability that easily allows for repeatability, which minimizes the variation between technicians (internal) and programs (external)? 4) $\mathbf{L N}_{2}$ storage capacity-Can the devices be easily and safely handled and identified? Is their storage potential space efficient? Does the device offer security and safety from physical damage or possible contaminants as an aseptic closed system? 5) Recovery potential /Survivability_ls the device design prone to potential problems in the guaranteed recovery of embryos, and will they reliably vitrify and maintain complete cellular integrity post-warming? The latter specific quality concern, the recovery rate, has actually been surprisingly minimized in published reports; this is done by generally hiding the unfavorable outcome (i.e., lost embryo or egg) in typically-good survival rates. Any device prone to inconsistent recovery $(<99 \%)$ is seriously flawed and constitutes a procedural liability.

Our aseptic, closed $\mu$ S-VTF method has been strategically developed to account for each quality-control measure. However, after 5 years of superior clinical success and validation ${ }^{14}$, the procedure had to be modified. The original $0.3-\mathrm{mL}$ embryo straws (possessing a hydrophobic plug) were removed from the IVF industry and replaced with a $0.3-\mathrm{mL}$ semen straw possessing a standard cotton/PVP plug (i.e., relabeled as a semen/embryo straw). This procedural paper outlines the specific steps and strategies needed to implement $\mu$ S-VTF safely, simply, and effectively. Furthermore, we highlight the modification(s) needed to reliably account for supply limitations, until such time as an alternative ideal straw container is reintroduced back into the clinical laboratory.

\section{Protocol}

The development of the $\mu$ S-VTF procedure was conducted as part of an approved Institutional Review Board (IRB) study in 2008-2009 (Aspire IRB, Santee, CA) on human oocyte and embryo cryopreservation. The procedure has since been routinely applied to the clinical treatment of infertility patients who have signed informed consent forms.

\section{Quality-control Considerations}

1. Use different colors (e.g., pens, labels, or identification (ID) rods or plugs) to distinguish patients if cryopreserving more than one group of embryos in a day.

2. Use different dishes and pipettes for each patient. Minimize the volume of medium transferred when moving the blastocysts from one solution to another.

3. Cryopreserve one blastocyst per straw or device. Maintain aseptic/sterile technique and adhere to all safety precautions.

4. Confirm the Physicians' Referral Form and signed patient consent before initiating the procedures. Check the disposition of the patients' embryo(s) in inventory.

5. Use a "Release of Liability and Consent for Transport" if the blastocysts are to be transferred to another facility. Ensure that all signatures are witnessed onsite or notarized.

6. Confirm proper quality-control measures for the medium and the protein supplements, as appropriate, including bioassays, endotoxin determinations, and expiration/lot number verification and tracking.

7. Establish critical limits (i.e., low levels of concern) for clinical cryopreservation outcome monitoring and evaluation, such as recovery rates: < $95 \%$, survival rates: $<80 \%$, and clinical pregnancy rates using single-euploid blastocysts: $<50 \%$.

\section{Cryopreservation Procedure}

1. Preparation

1. Complete the appropriate paperwork, including entering data into the worksheets and the cryopreservation database and inventory $\log (\mathrm{s})$.

2. Prepare fresh cryopreservation solutions or use a commercial source (abiding by the manufacturer's recommended storage and shelflife conditions).

3. Straw preparation.

1. Open the labeling side of the individualized sterile packets. Detach the filling nozzle found on the traditional 0.3-mL embryo straw by grasping it against the packaging and partially lifting and pulling the straw out; this will expose the label end to ambient conditions while the straw rests aseptically inside the plastic package.

1. (Modification) For $0.3-\mathrm{mL}$ semen/embryo straws, push the plug down $0.5 \mathrm{~cm}$. Apply an internal seal in front of the cottonPVP plug through use of a basic tabletop impulse sealer. 
2. Set the straw onto the Teflon surface of the electrode, just in front of the plug. Depress the handle to activate the heat. Release the handle when the red light appears. To ensure a complete weld seal, heat at a temperature that sufficiently flattens the opposing surfaces, then flip it over $180^{\circ}$ and reseal the opposite surface ${ }^{19}$ as needed.

4. Using a cryomarker, label each straw/device and cryogoblet with the patient name, a unique ID number (e.g., SSN, DOB, or patient ID number), and the date of freezing. Also include a unique number on each straw/device (e.g., 1, 2, 3, etc.).

1. Secure the label (preferably colored) onto a color-weighted ID rod to be inserted and sealed into a 0.3-mL embryo straw. Return the straw to its individual sterile package until use.

Note: If only $30-\mathrm{mm}$ color-weighted ID rods are available, then the additional insertion of two ball bearings is needed, adjacent to each side of the ID rod.

5. Identify each cane with a unique inscribed ID number. Identify a $\mathrm{LN}_{2}$ storage tank number and a canister number where each patient's specimen(s) can be stored long-term.

6. Prepare fresh cryo dishes $(100 \mathrm{~mm})$ by labeling the bottom surface with the patient name, solution ID, and embryo/holding droplet ID (Figure 1A). Specifically, set up 4 rows of droplets: isotonic hepes-buffered medium (top), V1 (equilibration solution (ES)), V2 (intermediate solution (IS)), V3 (vitrification solution (VS); bottom). Write the embryo ID on the upper-right side with labeled columns. Note: A series of 25 - to $50-\mu \mathrm{L}$ wash droplets $(n=3)$ is used to ensure that each individual final $10-$ to $15-\mu \mathrm{L}$ holding droplet/solution type (i.e., V1, V2, or V3) is pure and undiluted. Using this approach, up to five individual embryos can by vitrified using a single dish. Keep each dish covered when not in use to avoid any room temperature evaporation/dehydration of the droplets.

7. Prepare sterile flexipettes by cutting $3 \mathrm{~cm}$ off their base end (referred to as "VTF tips"). Insert them onto individual pipettes (set at $3 \mu \mathrm{L}$ ) and set the pipette-VTF tips upright in a styrofoam tube rack (Figure 1B).

2. Embryo Selection

1. Confirm patient/specimen identification.

2. Using an inverted microscope (200 - 400X magnification), grade and classify the blastocysts according to the Gardner grading system $^{20}$, using a numeric classification of 1 to 6 for early to hatched blastocysts, respectively, and an A, B, or C grade assigned to the inner cell mass (ICM) and trophectoderm (TE).

Note: In preparation for blastocyst biopsy and euploidy analysis, the zona pellucida is pre-hatched by laser ablation on Day 3 to promote the early herniation of the TE and thus requires a modified grading classification ${ }^{2}$. In brief, Grade 3 full blastocysts exhibit up to $10 \%$ TE herniation, Grade 4 expanded blastocysts are $10-50 \%$ hatched, and Grade 5 hatching blastocysts have $>50 \%$ TE extrusion (Figure 2A-C).

Note: Our preference is to cryopreserve Grade 3 or higher blastocysts of $\geq B B$ quality on Day 5 or 6 . However, vitrified blastocysts of lower quality (C) and post-compaction embryos at earlier stages (including late morula to Grade 1 or 2 blastocysts) can certainly survive warming completely intact and yield viable embryos capable of achieving live births.

3. Collapse the blastocoele of each blastocyst prior to the onset of the cryodilution process by micropuncturing a trophectoderm junction or by performing a laser pulse ablation of a trophectodermal cell ${ }^{21}$ if using low-molarity vitrification solutions (<6.5 M or $\left.40 \% \mathrm{v} / \mathrm{v}\right)$. Note: The need to collapse the blastocysts is not device dependent. In our own early development of $\mu S-V T F(2008)$, we initially used ethylene glycol (EG)/DMSO solutions successfully with oocytes (1 out of 1 full-term pregnancy), but a sub-optimal (<80\%) survival/ development of mouse blastocysts ${ }^{16}$ was experienced by others ${ }^{21}$. Since high molar glycerol-based solutions (exceeding $\left.7.9 \mathrm{M}\right)$ are used in our current VTF system (since 2009), physical blastocoele collapse is unnecessary. However, the selective hatching of hatched blastocysts is advisable in general.

3. Cryodilution and Blastocyst Loading

1. Remove the patient culture dish from the incubator and confirm patient/specimen identification with another person (i.e., witnessed by a secondary source) before removing the embryo(s) to be vitrified.

2. Confirm blastocyst development per step 2.2.2, update the cryo data sheet, and, under stereomicroscopy, pipette blastocyst(s) from the culture dish into an isotonic holding droplet of the cryo dish.

3. Move each blastocyst into individual holding droplets using the vitrification flexipette (i.e., the VTF tip).

4. Move each blastocyst into V1 (ES) solution.

1. Prefill the flexipette with $\mathrm{V} 1$ solution $(3 \mu \mathrm{L})$ and place half the contents onto the embryo.

2. Aspirate the embryo and move to the first of three $\mathrm{V} 1$ wash droplets (referred to in step 2.1.6), pipette the embryo 2 - 3 times around perimeter, and clear the pipette contents in the droplet (avoiding bubble formation) or outside on the dish surface.

5. Fill the VTF tip with the next V1 wash drop and repeat the aspiration of the embryo(s). Repeat a third time and move the blastocyst(s) to an individual V1 holding drop. The dilution/washing steps should take 30 to $45 \mathrm{~s}$. Pre-load each VTF tip with the next solution (e.g., V2), insert the pipette in a rack, and use the next pipette (according to the setup in step 2.1.7) (Figure 1B).

Note: Since the total exposure time in non-dimethyl sulfoxide (DMSO) containing V1 and V2 solutions is 5 min each, the pipetting of individual blastocysts should be evenly staggered within that interval, considering that the final V3 step will require at least 1 min per blastocyst to perform. For example, if there are 4 blastocysts in a vitrification group, pipette blastocysts \#1-2-3-4 at 75-s intervals. Note: The Innovative Cryo Enterprises (ICE) dilution protocol described above is distinctly different than most other commercial vitrification procedures, which generally involve DMSO-containing solutions. Those protocols achieve the same stepwise dilutions through a gradual, but less controlled, merging of wash solution (i.e., isotonic medium), ES, and VS.

6. Repeat steps 2.3.4 and 2.3.5 using V2 (IS) solutions.

7. Repeat steps 2.3.4 and 2.3.5 using V3 (VS) solutions.

8. Upon placing each blastocyst in the V3 holding droplet, clear the residual solution and bubbles from the VTF tip (outside the droplet), and then fill the tip completely with clean V3 around the embryo. Expel approximately one-third of the volume $(1 \mu \mathrm{L})$ and pipette the blastocyst(s) and remaining V3 completely into the VTF tip.

9. Remove the VTF tip from the pipette, wipe the tip dry of residual V3 on the outer surface by using a sterile gauze pad, and insert the tip end-first into the open end of the pre-labeled straw. A "dry tip" is critical to preventing possible inner straw adherence. 
10. Invert the straw (label end down), observe the tip at the inner plug or seal, and safely seal the open end with $1 \mathrm{~cm}$ of air space. Preferably, use an automatic sealing device (e.g., Syms I) to eliminate technical variation.

Note: The advantage of using straws that are composed of an ionomeric resin plastic (e.g., HSV or $\mu$ S-VTF) is that using any properlyoperated heat sealing device creates a reliable "weld" seal, as noted in step 2.1.3.1.

11. Once sealed, plunge the closed straw directly into $\mathrm{LN}_{2}$ in a stainless steel Dewar flask and place the straw(s) into the open goblet attached to the patient's cane for storage.

Note: Because $\mu$ S-VTF straws use 40-mm weighted ID rods to offset the buoyancy of the air-filled straw, the use of inverted goblets or empty cryovials to cover the specimen(s) is not required unless transportation is involved.

4. Warming and Cryo-solution Elution/Dilution

1. Confirm the Physicians' Referral Form regarding vitrified embryo transfer date, scheduled time, and embryo details (number to thaw/ transfer, specific embryo number if PGS tested, etc.).

2. Prepare fresh warming solutions (1.0 M sucrose stock solution) and/or use a commercial source (recommended 1 week-1 month of shelf life once in use, $4{ }^{\circ} \mathrm{C}$ storage).

1. Aliquot $17.1 \mathrm{~g}$ of sucrose into $50-\mathrm{mL}$ sterile flasks upon initial opening (e.g., weekly supply source) due to the risks of endotoxin accumulation in sucrose granules upon repeated ambient exposure.

2. Mix pre-weighed granular sucrose into solution $(1.0 \mathrm{M}$ stock $=3.42 \mathrm{~g} / 10 \mathrm{~mL}$ of hepes-buffered human tubal fluid [H-HTF]) and warm the solution up to $37^{\circ} \mathrm{C}$ to increase the solubility of the granules. Repeatedly invert the container to help speed up and complete the final mixing.

Note: Filtration $(0.22-\mu \mathrm{m}$ filter) performed using positive pressure filtration units is recommended for viscous solutions (>0.5 M sucrose) or high volumes. Hand pressure pumps are sufficient and inexpensive, whereas an electrical pump is noisy, causes vibrations, and is simply not required.

3. Warm $\left(37^{\circ} \mathrm{C}\right)$ one $10-\mathrm{mL}$ tube each of $1.0 \mathrm{M}$ sucrose solution and $\mathrm{H}-\mathrm{HTF}$ medium per patient for $\mu \mathrm{S}-\mathrm{VTF}$ warming bath use.

4. Prepare fresh thawing dishes (6-well plate) by labeling the cover surface with the patient name and the solution IDs.

Note: In this case, we used: (1) T1 wash $(200 \mu \mathrm{L}$ without oil overlay, (2) T1 (100 $\mu \mathrm{L})$ with $1 \mathrm{~mL}$ of oil, (3) T2 (100 $\mu \mathrm{L})$ with oil, (4) T3 $(100 \mu \mathrm{L})$ with oil, (5) T4 (100 $\mu \mathrm{L})$ with oil, and (6) T5/200 $\mu \mathrm{L}$ isotonic HEPES-buffered medium with $10 \%$ human serum albumin (HSA) or $20 \%$ serum substitute without an oil overlay. Apply a universal sucrose solution step-down protocol-T1 = $1.0 \mathrm{M}, \mathrm{T} 2=0.5$ $\mathrm{M}, \mathrm{T} 3=0.25 \mathrm{M}$, and T4 $=0.125 \mathrm{M}$-if the ICE or another commercial source is not available, as proposed for slow-frozen embryos ${ }^{22}$. Furthermore, note that the initial T1 wash and the final T5 equilibrations can be performed in 30-mm Petri dishes and the 4-well dish used for steps 2-5 above, involving an oil overlay.

5. Thaw only one patient's embryo(s) at a time.

1. Check the Physicians' Referral Form and confirm the cycle before warming the indicated number (i.e., 1 or 2 blastocysts); assess survival/probable viability by observing osmotic changes (i.e., shrinkage and rehydration) and intact membranes, and, if questionable, contact the physician and/or patient before warming another blastocyst.

6. Confirm patient/specimen identification with a co-worker (i.e., witnessed by a secondary source).

7. Place the patient cane in a Dewar flask filled with $\mathrm{LN}_{2}$ and isolate identify the straw to be warmed.

8. Bring the 6-well dilution dish to the center of the stereomicroscope stage. Placing a 60-mm Petri dish off to one side (i.e., depending on the left-hand or right-hand preference of the technician), add the warmed sucrose $(1.0 \mathrm{M})$ and $\mathrm{H}-\mathrm{HTF}$ solutions to create a $0.5 \mathrm{M}$ sucrose warming bath (uncovered).

Note: VTF tips are only warmed in $0.5 \mathrm{M}$ sucrose solutions as a QC safeguard, to insure the retention of embryo viability in the rare event that an embryo is expelled upon rapid warming 22 .

9. Hold the upper straw seal above liquid level to confirm the identity, and then grasp and secure the straw below the internal plug using Mayo scissors (the VTF tip should still be submerged in $\mathrm{LN}_{2}$ ).

1. Firmly tap the scissors on the Dewar flask (a couple times) to remove the VTF tip from the inner straw sidewall as a QC precaution; if the VTF tip is adherent, the tapping ensures that the tip base drops to the sealed end opposite the labeled end, thus providing an air space near the plug end.

10. Lift the straw with the scissors into ambient air in a horizontal position (next to or below the stereoscope surface) and grasp the nonlabeled end (label to the right side). Cut the straw at the inner plug or seal and lift it above the warming bath dish. Pour the VTF tip into the warm sucrose bath at a $60^{\circ}$ angle until it is completely extruded and has been rapidly warmed $\left(>6,000^{\circ} \mathrm{C} / \mathrm{min}\right)$; the base of the flexipette will rest above the dish sidewall.

1. Allow the VTF tip to free-fall into the bath. Gently tap the upper straw surface if the tip does not immediately emerge. If it only appears part way, assist in the extrusion by grasping the shaft of the flexipette with the scissors or fine forceps.

11. After $5-10 \mathrm{~s}$, grasp the pipette base and insert into a disposable pipetting device; a hole in the bulb acts to release pressure upon insertion. Cover the bulb hole with an index finger and gently squeeze the bulb to release the vitrified blastocyst into the T1 wash (\#1) while viewing by stereomicroscopy. Once confirmed, clear the residual V3 (VS) solution and bubbles by positive pressure, evacuating the contents into the center, unused discard well. Start a 5-min timer.

Note: All sucrose dilution steps are performed at room temperature $\left(21 \pm 2{ }^{\circ} \mathrm{C}\right)$.

12. Remove VTF tip and place it onto a pipette. Proceed by moving the embryo into T1 \#2 under oil for the remaining time (approximately 3 $-4 \min )$.

1. If a second blastocyst is required for transfer, immediately warm a second straw and VTF tip (repeating steps $2.4 .9-2.4 .11$ ) from the patient before initiating step 2.4.12. Move both blastocysts together for a minimum elution in T1 (1.0 M sucrose) of at least 3 $\min$.

13. Pre-fill the flexipette with the next solution, and then move and dilute the blastocyst(s) in T2, T3, and T4 at 3-min intervals. If the zona pellucida has not been previously laser ablated (i.e., hatched), do so in T4. Place the dish onto the stage of an inverted microscope and image the embryo on a computer monitor. Align the zona within a designated red circle and activate 2 or 3 impulses of a diode laser (beginning at the edge of the perivitelline space and moving outward) to create an opening ${ }^{2,19}$. 
14. Equilibrate the blastocysts in T5 (i.e., isotonic medium) for 5 min on a warm surface $\left(37^{\circ} \mathrm{C}\right)$.

15. Assess the initial embryo survival based on the predominant presence of intact cell membranes with even, translucent cytoplasms devoid of pyknotic darkening. Place the embryo(s) into culture for 2 - $6 \mathrm{~h}$ before embryo transfer, at which time, the embryo development/survival should be re-evaluated and documented.

Note: If more than 1 viable blastocyst exists at the time of transfer and the patient opts to proceed with only 1 blastocyst, the supplemental blastocyst can then be successfully re-vitrified (rVTF) by repeating steps 2.3.3 - 2.3.11. We have several confirmed live births following single rVTF events.

Note: rVTF has been experimentally performed up to 5 times without a significant effect on the survival/viability of aneuploidy human blastocysts vitrified in a metastable glycerol-based solution.

\section{Representative Results}

1,341 vitrified blastocysts were warmed between 2012 to June 2014, and 1,341 embryos recovered (100\%) while 1,316 survived (98.1\%). Biopsied blastocysts experienced over $99.5 \%$ survival. Upon transferring predominantly single, genetically tested, euploid, vitrified embryos, we were able to achieve some of the highest implantation rates and live birth rates in the USA, independent of age (Figure 3$)^{20}$, according to recent national statistics reported by the Center for Disease Control and the Society for Assisted Reproductive Technologies (Tables 1 and 2 ). Evaluating a good prognosis patient population (less than 35 years old) for cryopreserved cycles alone (Table 1), our laboratory outperformed the national average for both implantation rates (i.e., the efficiency of an embryo to establish a pregnancy) and ultimately live birth rates by over $30 \%$. Additionally, the initial validation/verification of our modified storage straws in mid-2014 using 70 research-consented, re-vitrified aneuploid blastocysts resulted in $100 \%$ recovery and $100 \%$ survival.

A

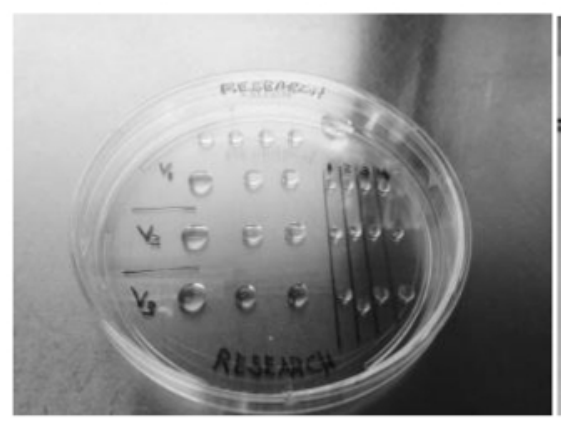

B

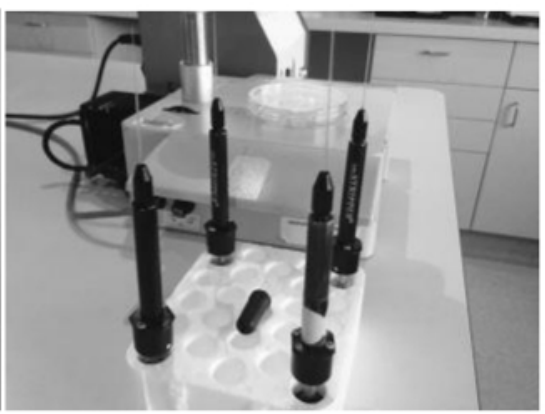

Figure 1. MicroSecure VTF Setup. The VTF dish $(\mathbf{A})$ is assembled with 3 distinct rows of vitrification solutions, which utilize 3 wash droplets before placement in distinct, numbered holding droplets. Additionally, individual pipettes with shortened VTF tips (i.e., 300- $\mu \mathrm{m}$ ID flexipettes) are secured and organized in a styrofoam tube rack (B), which can be rotated for orderly use. Note that a representative disposable micropipette bulb pipetting assembly is resting on the styrofoam rack. Please click here to view a larger version of this figure.

A

B

C

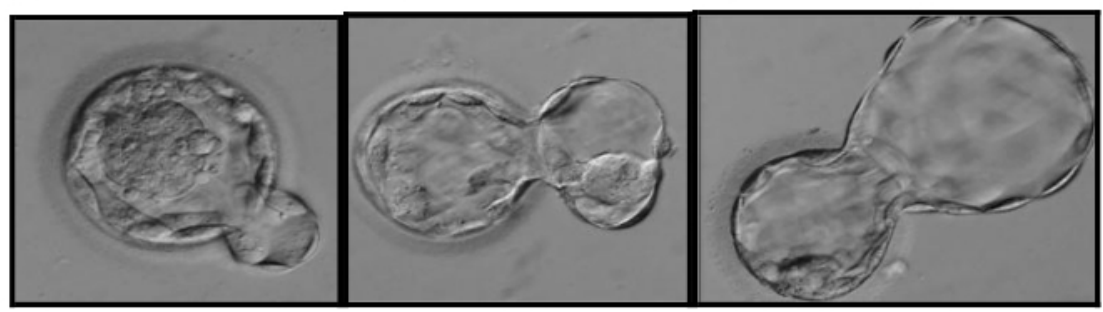

Figure 2. Modified Blastocyst Grading System. Our modified Gardner blastocyst grading system accounts for the induced herniation of TE cells to facilitate blastocyst biopsy. The modification considers the premature hatching of full blastocysts (A: Grade $3=$ less than $10 \%$ herniation) and expanded blastocysts (B: Grade $4=$ up to $50 \%$ herniation), while a hatching blastocyst (C) has greater than $50 \%$ herniation ${ }^{16}$. Please click here to view a larger version of this figure. 


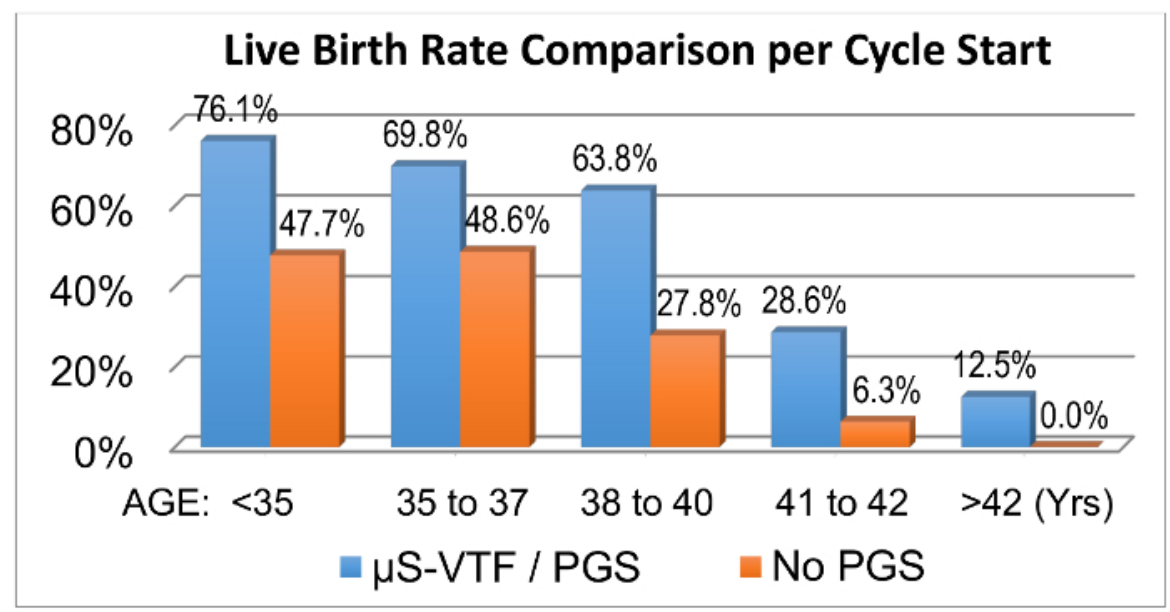

Figure 3. Comparative Pregnancy Outcomes. Over a cumulative 1.5-year interval, pregnancy data were compared to assess the effects of transferring vitrified-warmed euploid blastocysts $(n=172$ cycles $/ 144$ transfers) compared to non-PGS cycles $(n=160$ cycles $/ 153$ transfers) involving predominantly fresh transfers ${ }^{18}$. For our experimental purposes, this data clearly reveals that biopsied blastocysts vitrified by the $\mu S-$ VTF procedure maintain their viability. Please click here to view a larger version of this figure.

\begin{tabular}{|l|l|l|l|l|}
\hline Data Source & \# Cycles & $\begin{array}{l}\text { Mean \# of embryos } \\
\text { transferred }\end{array}$ & Implantation Rates (\%) & Live Birth Rates (\%) \\
\hline NB Lab * & 144 & 1.2 & $74.00 \%$ & $74.50 \%$ \\
\hline National Average & 20,423 & 1.7 & $39.60 \%$ & $44.10 \%$ \\
\hline $\begin{array}{l}\text { * Data were averaged based on the 2014 performance of Orange County Fertility, Southern California Fertility Center and the Southern California } \\
\text { Center for Reproductive Medicine clinics using the current Ovation Fertility laboratory (NB Lab) which founded the microSecure vitrification } \\
\text { procedure in 2008. }\end{array}$
\end{tabular}

Table 1. 2014 CDC Assisted Reproductive Statistics. Frozen embryo transfer pregnancy data of women under 35 years old from three reporting physician clinics using our NB Lab compared to the US 2013 national average of over 450 reporting clinics.

\begin{tabular}{|l|l|l|l|l|}
\hline SART National Averages & & & \\
\hline Clinic - State & Women & Women & Women & Women \\
\hline & $<35$ yrs & $35-37$ yrs & $38-40$ yrs & $41-42$ yrs \\
\hline SCCRM-CA * & $63.1 \%$ & $58.3 \%$ & $40.6 \%$ & $32.3 \%$ \\
\hline CCRM-CO * & $64.7 \%$ & $61.5 \%$ & $40.4 \%$ & $32.2 \%$ \\
\hline RMA-NJ * & $63.2 \%$ & $59.7 \%$ & $34.6 \%$ & $18.7 \%$ \\
\hline National Average & $48.6 \%$ & $38.3 \%$ & $12.3 \%$ \\
\hline $\begin{array}{l}\text { SCCRM-Southern California Center for Reproductive Medicine; CCRM-Colorado Center for for Reproductive Medicine; and RMA- } \\
\text { Reproductive Medicine Associates }\end{array}$ & \\
\hline $\begin{array}{l}\text { * These leading clinics have all predominantly implemented vitrified embryo transfer cycles, in association with preimplantation genetic testing, } \\
\text { into their standard practice of patient care to optimize live birth success per embryo transplanted. }\end{array}$ \\
\hline
\end{tabular}

Table 2. 2014 Cumulative live birth rates, as reported by the Society for Assisted Reproductive Technologies (SART), for the SCCRM Clinic using our Ovation Fertility Lab in Newport Beach, CA, contrasted with several other respected programs, as well as with the SART national averages.

\section{Discussion}

Today, there is a high expectation of attaining complete blastocyst survival (>95\%) and achieving implantation success similar to that of fresh embryos. Some groups have suggested that the live birth rates of vitrified embryo transfer cycles are perhaps even higher than fresh blastocysts when the intact cryopreserved embryo is transplanted into a healthy, non-hormonally-stimulated uterus. Our data clearly indicate that vitrification effectively and reliably maintains the viability of the fresh embryo. Furthermore, we have proven that our aseptic, closed method, called MicroSecure Vitrification ( $\mu \mathrm{S}-\mathrm{VTF}$ ), can achieve an optimal outcome comparable to or greater than the commercial standards (i.e., open device systems) used in the IVF industry.

Variation is associated with technical repeatability and reliability between individuals using a multitude of vitrification devices/methods. In turn, this has resulted in inconsistencies between programs applying vitrification. Therefore, it is not surprising that device familiarity is an important factor regarding laboratory proficiency and successful outcomes. It is this concept of "technical signature" ${ }^{11}$ that explains why repeatability between programs may be problematic. Not only has the development of more than a dozen commercial devices complicated this phenomenon, 
it has created quality-control concerns. Fortunately, closed vitrification systems, like $\mu$ S-VTF, Rapid-I, and VitriSafe, are now proving to be equally effective to open device systems ${ }^{12,13,14}$.

MicroSecure VTF is a novel, aseptic vitrification technique developed with technical ease, reliability, and cryo-security in mind. By combining the use of two previously-approved, FDA-compliant devices, it is a non-commercial vitrification system with the distinct advantage of having an established low cost, in contrast to specialized devices. In addition, its unique tamperproof and internalized dual-colored labeling system, as well as numerous other quality-control advantages, have made $\mu$ S-VTF an attractive global option ${ }^{11}$. As a non-commercial VTF device, however, its widespread industry application is evolving slowly. Only through the continued publication of its safety, security, and clinical effectiveness will $\mu \mathrm{S}-$ VTF gain growing utilization.

In August 2014, when faced with the inability to acquire the original 0.3-mL embryo straw manufactured with an inner, non-wicking hydrophobic plug, we were able to reliably modify the $\mu S$-VTF method. In short, the new $0.3-\mathrm{mL}$ semen/embryo straws with their cotton-PVP plugs were effectively adapted. This was simply achieved by creating an inner seal before the cotton plug, thus preventing contact and fluid wicking with the open-ended VTF tip (i.e., the flexipette containing the embryo or eggs). Furthermore, if $40-\mathrm{mm}$ ID rods are not accessible, the buoyancy issue associated with the lighter $30-\mathrm{mm}$ rods can be counterweighted using two ball bearings.

These additional steps have made the technique slightly less simple, but still highly effective. Today, economics and efficacy are becoming increasingly important concerns, as biopsied blastocysts are typically vitrified individually until their euploidy status is confirmed on a genetics report. Blastocysts with a confirmed non-viable aneuploidy status typically get discarded, with patient consent, within weeks. Thus, a majority (> $50 \%$ ) of VTF devices are discarded after short-term storage, causing an escalating annual cost when using commercial devices. Overall, $\mu \mathrm{S}-$ VTF is a highly-effective, reliable, and repeatable procedure for the cryopreservation of human blastocysts. The superior quality-control design securely labels and safely stores embryos/oocytes while eliminating recovery failure and optimizing post-warming survival and viability, which justifies the system's use as a universal approach to embryo vitrification.

\section{Disclosures}

M.C. Schiewe serves as a member of the scientific advisory board to Innovative Cryo Enterprises and as a Technical Director to the California Cryobank. Production of this video-article has been paid for by Ovation Fertility. The authors have no competing financial agreements or conflicts of interests to disclose regarding the vitrification of gametes and embryos.

\section{Acknowledgements}

M.C. Schiewe would like to thank Mr. Forest Garner at the Fertility Center of Las Vegas for his statistical expertise in analyzing and evaluating annual CDC and SART data. Also, the authors wish to thank their Medical Director, Dr. Robert E. Anderson, for his dedicated support and faith in our technical abilities and expertise.

\section{References}

1. Edgar, D.H., \& Gook, D.A. A critical appraisal of cryopreservation (slow cooling versus vitrification) of human oocytes and embryos. Hum. Reprod. Update. 16, 536-554 (2012).

2. Schiewe, M.C., Whitney, J.B., \& Anderson, R.E. Potential risk of monochorionic dizygotic twin blastocyst formation associated with early laser zona dissection of group cultured embryos. Fertil. Steril. 103, 417-21 (2015).

3. Schiewe, M.C. The historic development and incorporation of four assisted reproductive technologies shaping today's IVF industry. J. Fertil. In Vitro Reprod. Med. Genet. 4, 2-9 (2016).

4. Rall, W.F., \& Fahy, G.M. Ice-free cryopreservation of mouse embryos at -196 degrees $C$ by vitrification. Nature. 313, $573-575$ (1985).

5. Rall, W.F., Wood, M.J., Kirby, C., \& Whittingham, D.G. Development of mouse embryos cryopreserved by vitrification. J. Reprod. Fertil. 80, 499-504 (1987).

6. Schiewe, M.C., Rall, W.F., Stuart, L.D., \& Wildt, D.E. Analysis of cryoprotectant, cooling rate and in situ straw dilution using conventional freezing or vitrification for cryopreserving sheep embryos. Theriogenology. 36, 279-293 (1991).

7. Lane, M., Schoolcraft, W.B., \& Gardner, D. Vitrification of mouse and human blastocysts using a novel cryoloop container-less technique. Fertil. Steril. 72, 1073-1078 (1999).

8. Mukaida, T., Oka, C., Goto, T., \& Takahashi, K. Artificial shrinkage of blastocoeles using either a micro-needle or a laser pulse prior to the cooling steps of vitrification improves survival rate and pregnancy outcome of vitrified human blastocysts. Hum. Reprod. 21, 3246-3252 (2006).

9. Kuwayama, M., Vatja, G., leda, S., \& Kato, O. Comparison of open and closed methods for vitrification of human embryos and elimination of potential contamination. Reprod. Biomed. Online. 11, 608-614 (2005).

10. Vanderzwalmen, P., et al. Births after vitrification at morula and blastocyst stages: effect of artificial reduction of the blastocoelic cavity before vitrification. Hum. Reprod. 17, 744-751 (2002).

11. Schiewe, M.C. Quality control factors influencing the successful and reliable implementation of oocyte and embryo vitrification. In: MarcoJiménez, $\mathrm{F}$ and Akdemir Koç, $\mathrm{H}$ (eds.) Cryopreservation. $\left(1^{\mathrm{st}} \mathrm{Ed}\right.$.). InTech. Rijeka, Croatia, (2016).

12. Stachecki, J.J., Garrisi, J., Sabino, S., Caetano, J.P.J., Wiemer, K., \& Cohen, J. A new safe, simple, and successful vitrification method for bovine and human blastocysts. Reprod. Biomed. Online. 17, 360-67 (2008).

13. Panagiotidis, Y., et al. Open versus closed vitrification of blastocysts from an oocyte-donation programme: a prospective randomized study. Reprod. Biomed. Online. 26, 470-476 (2013).

14. Papatheodorou, P. et al. Open versus closed oocyte vitrification system: a prospective randomized study. Reprod. Biomed. Online. 26, 595-602 (2013). 
15. Schiewe, M.C., Zozula, S., Anderson, R.E., \& Fahy, G.M.Validation of microSecure vitrification ( $\mu$ S-VTF) for the effective cryopreservation of human embryos and oocytes. Cryobiology. 71, 264-72 (2015).

16. Schiewe, M.C. MicroSecure vitrification for oocytes and embryos: optimum simplicity, security and cost and effectiveness combining FDAapproved products. J. Clin. Embryol. 13, 33-51 (2010).

17. Rall, W.F. Factors affecting the survival of mouse embryos cryopreserved by vitrification. Cryobiology. 24, 387-402 (1987).

18. Seki, S., \& Mazur, P. Effect of warming rate on the survival of vitrified mouse oocytes and on the recrystallization of intracellular ice. Biol. Reprod. 79, 727-737 (2008).

19. Schiewe, M.C. General hygiene and quality control practices in an embryo-production laboratory. In: Stringfellow,DA and Givens, D (eds.) Manual of the International Embryo Transfer Society: A ProceduralGuide and General Information for the Use of Embryo Transfer Technology, EmphasizingSanitary Precautions. (3rd Ed.). International Embryo Transfer Society. Champaign, IL, (2008).

20. Whitney, J.B., Nugent, N.L., Anderson, R.E., \& Schiewe, M.C. Single center validation of routine blastocyst biopsy implementation. J. Asst. Reprod. Genet. (2016).

21. Liebermann, L., \& Conaghan, J. Artificial collapse prior blastocyst vitrification: improvement of clinical outcomes. J. Clin. Embryol. 16, 107-118 (2014).

22. Parmegiani, L., Tatone, C., Cognigni, G.E., Bernardi, S., Troilo, E., Arnone, A., Maccarini, A.M., Di Emidio, G., Vitti, M., Filicori, M. Rapid warming increases survival of slow-frozen sibling oocytes: a step towards a single warming procedure irrespective of the freezing protocol? Reprod Biomed Online. (2014). 